\title{
Die Zweiteilung der Amerikas im 19. Jahrhundert
}

Um das sich verändernde Verhältnis Europas zu den Amerikas im Norden und den Amerikas im Süden genauer untersuchen und um philosophisch-literarische Entwicklungen daraus ableiten zu können, ist es sicherlich angebracht, bevorzugt auf Reiseberichte aus jenem Zeitraum zurückzugreifen, den wir mit Gertrudis Gómez de Avellaneda erreicht haben. In der Sattelzeit des langen 19. Jahrhunderts war, wie im einführenden Teil dieser Vorlesung besprochen, der Topos der „Historia magistra vitae“ bekanntlich abgelöst worden von einem offenen, zukunftsorientierten Zeithorizont, der die Zyklen früherer geschichtlicher Vorstellungen teilweise relativierte, teilweise hinter sich ließ. ${ }^{1}$ Innerhalb einer derart gewandelten Zeitvorstellung wurde auch das Reisen durch die Zeit vermittels eines Reisens durch den Raum möglich, konnten doch an einem anderen Ort gleichsam die früheren Zustandsformen der allgemeinen Entwicklung der Menschheit - so schien es - besichtigt werden, zumindest dann, wenn man von der einsträngigen und linearen Geschichte einer einzigen Menschheit ausging. Und eine solche Vorstellung mit dem europäischen Teil der Menschheit an der Spitze des Fortschritts dominierte in allen Ländern Europas.

So versuchte bereits in der zweiten Hälfte des 17. Jahrhunderts Jean-Baptiste $\mathrm{Du}$ Tertre $^{2}$ insbesondere in seiner vierbändigen Histoire générale des Antilles habitées par les François (1667-1671), seinen Überlegungen zum „bon sauvage“, Erkenntnisse über die Entwicklung des Menschengeschlechts ausgehend von Beobachtungen in der Fremde zu gewinnen. So wurde es bereits in einer frühen Form neuzeitlichen Denkens möglich, Wissen über die Vorgeschichte des Eigenen durch eine Art rückwärtsgerichtete Zeitreise zu erhalten, sich mithin auf der Zeitskala der allgemeinen Geschichte der Menschheit vermittels gezielter Reisen zu bewegen. Im Anschluss an den Pater Fritz und andere intertextuelle Bezugstexte entfaltete der uns bereits bekannte Kubaner Alejo Carpentier dieses Modell einer Zeitreise in den Tropen der Amerikas in seinem großartigen Roman Los pasos perdidos. Dort gelingt es einem Musikologen, die Spuren in eine längst vergangene Epoche der Menschheitsgeschichte in der Jetztzeit aufzunehmen und durch eine Bewegung im Raum die gezielte Bewegung in der Zeit auszulösen. Noch in Claude

\footnotetext{
1 Vgl. hierzu Koselleck, Reinhart: Historia Magistra Vitae. Über die Auflösung des Topos im Horizont neuzeitlich bewegter Geschichte. In (ders.): Vergangene Zukunft. Zur Semantik geschichtlicher Zeiten. Frankfurt am Main: Suhrkamp 21984, S. 38-66.

2 Vgl. Funke, Hans-Günter: „Barbare cruel“ o „bon sauvage“? La funcionalización ambivalente de la imagen del indio en la „Histoire générale des Antilles“ (1667-1671) del Padre du Tertre. In: Dispositio (Ann Arbor) XVII, 42-43 (1992), S. 73-105.
}

Ә Open Access. () 2021 Ottmar Ette, publiziert von De Gruyter. (c) BY-NC-ND Dieses Werk ist lizensiert unter einer Creative Commons Namensnennung - Nicht-kommerziell - Keine Bearbeitung 4.0 International Lizenz. https://doi.org/10.1515/9783110703443-017 
Lévi-Strauss’ auf Brasilienaufenthalten des französischen Anthropologen in den dreißiger Jahren des 20. Jahrhunderts basierenden Tristes Tropiques lassen sich viele derartige Elemente einer Zeitreise zurück in die Anfänge des Menschengeschlechts herausarbeiten. Es handelt sich folglich um europäische Vorstellungen, die keineswegs nur in der Sattelzeit des 19. Jahrhunderts Bestand hatten.

Doch ist nicht allein die Reise rückwärts, sondern auch vorwärts in der Zeit möglich. Literarische Beispiele hierfür ließen sich leicht anhäufen und einen überaus einflussreichen Versuch einer derartigen Zeitreise in die Zukunft Europas durch eine Reise nach Amerika werden wir uns sogleich näher ansehen. Gleichzeitig kann auch die eigene Gegenwart durch die Beschäftigung mit dem Anderen als künftige Vergangenheit beleuchtet werden. Ein Beispiel hierfür - und ein aus Sicht des Gegenstandes unserer Vorlesung bis heute faszinierendes Exempel - ist das Werk des französischen Historikers und Politikers Alexis de Tocqueville, zu dessen Schaffen ich Ihnen gerne einige Biographeme und inhaltliche Einblicke vorab vermitteln möchte.

Alexis Charles Henri Clérel, Comte de Tocqueville wurde am 29. Juli 1805 in Verneuil-sur-Seine im Département Yvelines geboren und verstarb am 16. April 1859 im südfranzösischen Cannes. Aus einer adeligen Familie stammend, deren royalistisch gesinntes Oberhaupt in Verwaltung und Politik Frankreichs sehr aktiv war, wurde er nach dem Studium der Rechtswissenschaften in Paris 1826 zum Untersuchungsrichter in Versailles ernannt und machte später die Bekanntschaft von Gustave de Beaumont, mit dem er nach Amerika reisen sollte. Er hörte zu Paris die Geschichtsvorlesungen des berühmten François Guizot, die seine Auffassungen von Geschichte und Gesellschaft prägten, und wurde im Jahre 1830 promoviert.

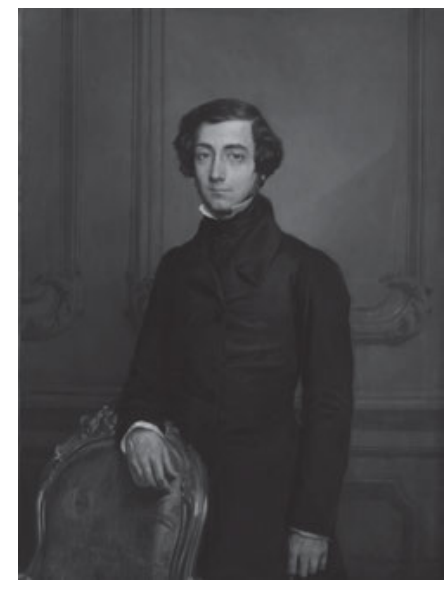

Abb. 41: Alexis de Tocqueville (Verneuil-sur-Seine, 1805 - Cannes, 1859). 
Auf Grundlage der von Mai 1831 bis Februar 1832 gemeinsam mit Beaumont unternommenen Reise in die Vereinigten Staaten von Amerika beruhte Tocquevilles Hauptwerk, das im Zentrum unseres Interesses an dem jungen Publizisten steht. Bei De la démocratie en Amérique - Sie bemerken, dass die USA in dieser Schrift bereits mit ,Amerika' gleichgesetzt werden - handelt es sich um ein geschichtliches und zugleich politisches Werk, in welchem der junge französische Adelige ausgehend von seinen Reiseerfahrungen die Demokratie in den USA und - so ließe sich sagen - zugleich die Zukunft Frankreichs und Europas zu beschreiben versuchte. Der erste Teil wurde 1835, der zweite 1840 veröffentlicht, fällt mithin genau in den Zeitraum, mit dem wir uns zuvor in Sab beschäftigt haben.

Die Grundthese seines Hauptwerks beruht darauf, dass der zum Zeitpunkt seiner Reise in die USA noch junge französische Historiker in der US-amerikanischen Demokratie seiner Zeit egalitäre Tendenzen verwirklicht sah, welche sich nach seinem Dafürhalten in Frankreich bereits abzeichneten und in Zukunft in ganz Europa durchsetzen würden. Sein Euvre stellte mithin in Raum und Zeit ein gemeinsames Spannungsfeld zwischen Alter und Neuer Welt her, gab diesem transatlantischen Bewegungsraum aber einen klaren historischen Zeitpfeil mit. Diese Vektorizität war eine wichtige Einsicht, wurden mit De la démocratie en Amérique doch die USA erstmals zum Schrittmacher einer politischen Entwicklung, der man auch in Europa früher oder später werde folgen müssen. Es handelt sich dabei um eine Vorstellung, welche zweifellos das gesamte 20. Jahrhundert dominieren sollte und bei einigen politischen Parteien in Europa wohl noch immer vorherrscht, aber insgesamt in unserer Epoche wohl um die Jahrtausendwende mehr als brüchig geworden ist.

Für Alexis de Tocqueville ging es vorrangig darum, die konkrete Verfassungswirklichkeit einer auf Egalitarismus gründenden Demokratie in den Vereinigten Staaten in ihren gesellschaftlichen und politischen Folgen einzuschätzen und dabei Vergleiche mit der französischen Geschichte mit Blick auf zukünftige Entwicklungen zu ziehen. Dabei interessierte er sich stark für das Prinzip der Volkssouveränität sowie für die von der staatlichen Struktur beförderten Chancen einer politischen und gesellschaftlichen Partizipation innerhalb eines demokratischen Systems, das nicht von einer Elite, sondern von einer Masse beherrscht werden sollte und wurde. Tocqueville sah die USA erstmals an der Spitze einer epochalen Entwicklung, welche die westlichen Gesellschaften des 19. und 20. Jahrhunderts prägen sollte. Die Reise in die USA war für ihn folglich nicht eine Reise in die Vergangenheit Europas, sondern ganz im Gegenteil eine Zeitreise in die Zukunft eines Europa, welche durch die Erfahrung der USA konkret beleuchtet und erhellt wurde.

Es ist aus heutiger Sicht überaus spannend, das von Tocqueville analysierte demokratische System und die historische Herausbildung der verfassungsmäßi- 
gen „Checks and Balances“ zu sehen, das in seiner Abhängigkeit von einer zunehmend plutokratischen politischen Spitze und in der eindeutig imperialistischen Ausrichtung am Ende des 19. Jahrhunderts von José Martí in seinen Escenas norteamericanas analytisch präzise beschrieben wurde. In Anknüpfung an Tocqueville sind dies Darstellungen und Analysen aus der Feder Martís, welche in Zeiten einer oligarchischen Bloßlegung dieses demokratischen Systems unter der Präsidentschaft Donald Trumps nachhaltig bestätigt wurden und ebenfalls nur wenig von ihrer Aktualität eingebüßt haben.

Alexis de Tocqueville interessierte sich besonders für die gesellschaftliche Elite des Landes angesichts des offensichtlichen Fehlens eines alteingesessenen Adelsstandes; ein Fehlen, mit dem der französische Adelige einen gewissen Niedergang geistiger Kultur verband. Doch richtete sich sein Interesse auch auf ein Parteiensystem, das grundlegend anders ausgerichtet war als in seiner französischen Heimat. Den unbezweifelbaren Vorzügen eines freiheitlichen Staatswesens stehen nach Ansicht von Tocqueville jene Gefahren gegenüber, welche von einer egalitären Masse und deren - heute würden wir sagen: populistischen - Meinungsschwankungen ausgehen könnten. Ich möchte betonen, dass Tocqueville von einer höchst konservativen Perspektivik aus sehr wohl jene Schwachpunkte eines demokratischen Systems ausmachte, die sich in unserer Zeit unter zweifellos grundlegend veränderten gesellschaftlichen und wirtschaftlichen Bedingungen als brandgefährlich erweisen sollten. Tocqueville erkannte dies mit scharfem Blick und warnte eindringlich vor Demagogen und Volksverhetzern, welche sich die offenkundigen Schwächen dieses Systems zu Nutze machen könnten.

Auch sonst sparte der französische Historiker bei aller Bewunderung nicht mit Kritik am demokratischen System der USA und seinen zyklischen Wahlperioden. Es ist aus heutiger Sicht beeindruckend, mit welcher Weitsicht und Scharfsichtigkeit er gesellschaftspolitische Entwicklungen kommen sah, die schon im 19. Jahrhundert virulent wurden, die aber auch noch in der Gegenwart die US-amerikanische Öffentlichkeit und ihre Medien in Aufregung halten oder gar erschüttern. Gewiss kann uns der elitäre und gegen die demokratische Masse gerichtete Gestus mancher Kritiken Tocquevilles heute nur fremd sein; doch seine kühl und analytisch vorgetragenen Kritikpunkte verdienen es in ihrer überwiegenden Mehrzahl, noch ernst genommen zu werden. Wenn wir sie mit jenen fundamentalen Kritikpunkten verbinden, welche ein José Martí vor dem Hintergrund des imperialistischen Ausgreifens der USA auf die Karibik, Mittel- und Südamerika entfaltete, dann können wir eine Vielzahl an Themen ausfindig machen, welche die damals künftige Entwicklung der Vereinigten Staaten durchaus sorgenvoll beleuchten.

Doch insgesamt ist Alexis de Tocqueville von der vereinigenden, große Teile der Bevölkerung zusammenführenden Kraft und Stärke des demokratischen Systems in den USA fest überzeugt. Aus globalpolitischem Blickwinkel erkannte 
Tocqueville in den Russen und in den US-Amerikanern jene beiden großen Völker und Länder, die schon bald Anspruch auf die Weltherrschaft erheben würden. Auch wenn diese weltpolitischen Prophezeiungen sich insgesamt im 20. Jahrhundert erfüllen sollten, lag der Schwerpunkt von De la démocratie en Amérique unverkennbar - wie im Titel des Werkes schon ausgedrückt - doch auf der Entfaltung des demokratischen Systems in den Vereinigten Staaten von Amerika und dessen Bedeutung für Europa. Am Beispiel der USA versuchte der Franzose zudem, vor künftigen Fehlentwicklungen der Demokratie in Frankreich und Europa zu warnen sowie Alarm zu schlagen, verstand seine Reise also auch als präventive Zeitreise, insoweit er in den USA Fehler und Fehlentwicklungen auszumachen suchte, die es künftig in seinem Heimatland - wie in Europa insgesamt - zu vermeiden gelte.

Eine Gefahr für die Demokratie erblickte Tocqueville vor allem in den aufstrebenden Magnaten der US-amerikanischen Industrieentwicklung, eine Prophezeiung, die zum Zeitpunkt José Martís längst Wirklichkeit geworden war, kritisierte der Kubaner doch ebenso eindringlich wie Tocqueville die oligarchischen und plutokratischen Strukturen, welche bis heute die demokratischen Grundlagen der USA bedrohen. Man übertreibt sicherlich nicht, stellt man Alexis de Tocqueville als einen feinsinnigen aristokratischen Anhänger eines demokratischen Systems in den USA dar, der frühzeitig vor den Gefahren einer Massendemokratie warnte, dessen Prophezeiungen sich auch in der „longue durée“ historisch bewahrheiteten und der noch heute eine kritische Sichtweise demokratischer Prozesse unter dem Einfluss oligarchischer und demagogischer Strukturen und Tendenzen entfaltet. Seine Reise in die Vereinigten Staaten von Amerika war eine Reise vorwärts in der Zeit: Er querte den transatlantischen Raum, um in die Zukunft zu gelangen.

Alexis de Tocqueville erkundete in seinem grundlegenden Werk jene Möglichkeiten, welche die demokratische Verfassung der Vereinigten Staaten für die europäischen Nationen und insbesondere für Frankreich bereithielt, welches also jene künftigen Aspekte seien, die man erhoffen dürfe oder befürchten müsse. ${ }^{3}$ Einen wichtigen Ausgangspunkt stellte dabei eine schlichte Frage dar: „Wohin führt unsere Reise?“ Wohin also entwickeln sich die Gesellschaften jenes Raumes, den man heute als den „Westen“ bezeichnet?

Glaubt man etwa, dass die Demokratie, nachdem sie den Feudalismus zerstört und die Könige besiegt hat, vor den Bürgern und den Reichen zurückweichen wird? Wird sie nun, da sie selbst so stark und ihre Gegner so schwach geworden sind, einfach stehenbleiben? Wohin gehen wir also? Niemand wüsste dies zu sagen; denn uns fehlen bereits die Ver-

3 Vgl. hierzu auch Neumeister, Sebastian: Alexis de Tocqueville. In: Lange, Wolf-Dieter (Hg.): Französische Literatur des 19. Jahrhunderts. Bd. II. Heidelberg: Quelle \& Meyer 1980, S. 85. 
gleichspunkte: Die Lebensumstände (conditions) sind heutzutage unter den Christen gleicher, als dies je zu einer anderen Zeit oder in einem andren Land der Welt der Fall war; so verhindert die Größe dessen, was schon getan ist, die Voraussage dessen, was noch getan werden kann. [...] Es tut nicht not, dass Gott selbst spricht, damit wir sichere Zeichen seines Willens erhalten; es genügt zu untersuchen, welches der gewöhnliche Gang der Natur und welches die beständige Tendenz der Ereignisse ist; [...]. ${ }^{4}$

Dies ist zu einem frühen Zeitpunkt die vollständig ausgebildete Einsicht in eine absolute Offenheit von Bewegungen in die Zukunft, einer Zukunftsoffenheit, die sich nicht mehr an vorgegebenen Modellen und Vorbildern zu orientieren vermag. Diese aufschlussreiche Passage mit der Frage danach, wohin die Reise einer spezifischen Gesellschaft, aber auch der Menschheit insgesamt gehen wird, ist folglich eine zentrale Ausdrucksweise der europäischen Moderne - und vielleicht mehr noch von Moderne überhaupt. Die epochenspezifische Erfahrung einer historischen Entwicklung, die sich zunehmend den bekannten Vorbildern entzieht und gerade im nachrevolutionären Frankreich der „Historia“ als „Magistra Vitae“ jegliche Legitimation abspricht, ${ }^{5}$ führt hier - die Formel „Où allons-nous donc?“ scheint es bereits anzudeuten - zu einer Ausweichbewegung im Raum: Eine Untersuchung der Demokratie in den Vereinigten Staaten soll Aufschluss geben über deren Entwicklung in Europa. Die Vereinigten Staaten von Amerika erscheinen als das Europa der Zukunft.

Damit wird die Reise gen Westen zu einer politischen Zeitmaschine, die Alexis de Tocqueville wohl als erster in einer langen, erst heute vermutlich definitiv abbrechenden Reihe von Reisenden in Gang setzte. Diese Reise gehorchte dem Motto: Reisen wir heute in die USA, um besser zu verstehen, was wir morgen in Europa leben und erleben werden! Sind deutsche oder italienische Reiseberichte der Nachkriegszeit über die Vereinigten Staaten in dieser Traditionslinie nicht auch häufig Erkundungsreisen gewesen, welche sich weniger um ein Verständnis aktueller Bedingungen des Fremden als um eine Reflexion der künftigen Möglichkeiten des Eigenen bemühten? Gerade nach dem Zweiten Weltkrieg häuften sich die Reisen europäischer Intellektueller, die in den Vereinigten Staaten untersuchen wollten, was die Herausforderungen von morgen für Europa sein werden.

So kann die Reise im Raum - ganz so, wie der kubanische Romancier Alejo Carpentier dies in seinem Orinoco-Roman Los pasos perdidos literarisch darstellte - zu einer Reise in verschiedenen Zeiten und zu verschiedenen Epochen

4 Tocqueville, Alexis de: De la démocratie en Amérique. Paris: Pagnerre 1848, Bd. I, S. 8. 5 Vgl. auch Koselleck, Reinhart: Historia Magistra Vitae. Über die Auflösung des Topos im Horizont neuzeitlich bewegter Geschichte. In (ders.): Vergangene Zukunft. Zur Semantik geschichtlicher Zeiten. Frankfurt am Main: Suhrkamp ${ }^{21984, ~ S . ~ 38-66 . ~}$ 
werden. Es handelt sich dabei um eine Reiseform, die ähnlich wie beim Umspringen der Utopie in die Uchronie, von der Raumebene auf die Zeitebene, dem Reisenden ab dem ausgehenden 18. Jahrhunderts die Möglichkeiten eröffnete, sich auf einer Raumebene tatsächlich durch die Zeit, die Zeitebene der gesamten Menschheit zu bewegen. Auch hier steht - nicht anders als in den nachfolgenden Jahrhunderten - Reiseliteratur für Literatur schlechthin ein. Der groß angelegte historiographische, politologische und soziologische Essay von Alexis de Tocqueville belegt dies auf eindrucksvolle Weise.

Die Offenheit der Zukunft führt mit Blick auf das von Tocqueville bereiste und zugleich uchronisch porträtierte Land im selben Atemzug zur Offenheit der Zukunft auch in einem technologischen, individuellen und auf die Erfüllung von Lebensprojekten gerichteten Zusammenhang. In diesem Zusammenhang gerät die literarische Form des Essays zu einer Suche nach neuen Lebensformen und Lebensnormen in einem prospektiven Sinne. ${ }^{6}$ Denn es beginnt sich ein neues Stereotyp herauszubilden, welches das Bild unserer Eltern in ganz Europa sehr nachhaltig mitgeprägt hat. Doch hören wir erst einmal den Verfasser dieses umfangreichen Versuchs über die Demokratie in Amerika. So heißt es im neunten Kapitel des dritten Bandes:

Die Amerikaner sind ein sehr altes und gebildetes Volk, welches ein neues und unermessliches Land gefunden hat, in welchem es sich nach Belieben ausbreiten und welches es mühelos befruchten kann. Dies ist in der Welt ohne Beispiel. In Amerika findet daher jeder Möglichkeiten, um sein Glück zu machen oder es zu vergrößern, die woanders unbekannt sind. Die Begehrlichkeit ist hier immer atemlos, und der menschliche Geist, beständig von Gelüsten der Imagination und intelligenten Vorhaben verführt, richtet sich ausschließlich auf die Verfolgung von Reichtum. Man sieht nicht nur in den Vereinigten Staaten wie in allen anderen Ländern in Industrie und Handel beschäftigte Klassen; vielmehr beschäftigen sich alle Menschen - und dies gab es zuvor noch nie - mit Industrie und Handel zugleich. ${ }^{7}$

In dieser Passage wird eine Vielzahl von Umbesetzungen zwischen der Alten und der Neuen Welt deutlich: Zum einen dürfen wir feststellen, dass dort, wo noch am Ausgang des 18. Jahrhunderts in der französischsprachigen Literatur der Begriff „Américains“ für die indigene Bevölkerung, also für die immer stärker marginalisierten und in Reservate zurückgedrängten Indianer reserviert gewesen war, nun der Begriff „Amerikaner“ ganz deutlich in jener Form auftritt, wie wir

6 Vgl. hierzu Ette, Ottmar (Hg.): Wissensformen und Wissensnormen des ZusammenLebens. Literatur - Kultur - Geschichte - Medien. Berlin - Boston: Walter de Gruyter 2012.

7 De Tocqueville, Alexis de: De la démocratie en Amérique, Bd. III, S. 68 f. 
ihn heute fälschlicherweise als ganz natürlich empfinden. In diesem Feld hat eine semantische Umbesetzung stattgefunden, in der die Selbstbezeichnung der „United States of America“ den Begriff „America“ selbst semantisch massiv reduziert und umgewendet hat - und nicht umsonst ist die marginalisierte indigene Bevölkerung daraus gänzlich verschwunden.

Wir sollten diese Tatsache als äußerst bedeutungsvoll herausarbeiten, ohne sie freilich nachzuahmen: In dieser Vorlesung wie auch in allen meinen Schriften steht der Begriff ,,amerikanisch“ für die gesamte Hemisphäre, so dass zu den „Amerikanern“ also ebenso die Bewohner des nördlichen wie des südlichen Teiles des amerikanischen Doppelkontinents gezählt werden. Und so wie wir die Bewohner des Südens als Lateinamerikaner benennen dürfen, müssen wir die Einwohner des nördlichen Amerika als Kanadier, US-Amerikaner und Mexikaner bezeichnen. Dass eine solche Bezeichnung derzeit nicht den Usancen entspricht, können Sie bereits an der völlig unwissenschaftlichen Bezeichnung ,Amerikanistik‘ ablesen. Eine derartige, bestenfalls umgangssprachlich akzeptable Benennung ist abzulehnen - zumindest dort, wo darunter nicht eine Wissenschaft gemeint ist, die sich mit dem gesamten amerikanischen Kontinent, seinen Sprachen, Geschichten, Kulturen oder Literaturen, beschäftigt.

Wenn in der obigen Passage aus der Feder von Alexis de Tocqueville die ,Amerikaner‘ als sehr altes und sehr aufgeklärtes Volk bezeichnet werden, dann bedeutet dies letztlich nur - und nichts anderes -, dass sie durch die Traditionen des Abendlandes einschließlich der Aufklärung mit Europa, mit den Europäern auf eine ebenso lange wie intime Weise verbunden sind. Die Anerkennung dieses Status als Kulturvolk beruht für den Franzosen folglich allein auf der Zurechnung der US-Amerikaner zu den Völkern des Abendlands. Die ,anderen“ Amerikaner, die indigenen Ureinwohner spielen in diesem Diskurs längst keine Rolle mehr: Sie sind aus der Geschichte und dem weiteren Fortgang der Weltgeschichte bereits getilgt und werden - ebenso wie der gesamte Kontinent - einfach wegeskamotiert. Ich sage dies nicht mit Schaum vor dem Mund: Aber bitte denken Sie das nächste Mal daran, wenn sie von den „Vereinigten Staaten von Amerika“ oder einer „American Academy“ in Berlin hören, dass es da noch , andere“ Amerikaner gibt!

Des Weiteren zeigt sich in dieser Passage überdeutlich und auf eine bedrückende Weise, dass ,Amerika“ als gleichsam leeres Land erscheint, so als würden jene Menschen in keiner Weise zählen, welche diese „immense“, unermessliche Region zuvor bewohnt hatten. Alexis de Tocqueville folgt hier nur jener Sprachregelung, die spätestens seit der Unabhängigkeit der Vereinigten Staaten vorherrschend war. Auf Grund dieser ,Menschenleere‘ könne dieses Land den Neuankömmlingen und seinen westlichen Bewohnern alle Möglichkeiten und mehr offerieren. ,Amerika' erscheint in diesem Zusammenhang bereits als Land der 
unbegrenzten Möglichkeiten, ein Topos, der auch in unseren Zeiten noch stereotyp wiederholt wird und Ihnen nicht unbekannt sein dürfte. In diesem Land der unbegrenzten Möglichkeiten aber spielen - und dies ist ein dritter gedanklicher Schritt - Industrie und Handel die zentrale Rolle. So entwickelt sich jene Sichtweise eines ganz auf Industrieproduktion und Handel ausgerichteten Volkes und einer Nation, die in ihrem ungestümen Vorwärtsstreben nach Perfektibilität - ich komme auf diesen Begriff zurück - letztlich anzeigen kann, wie sich das hinter den USA zurückfallende Europa künftig entwickeln wird.

Diese Denkrichtung wird schon wenige abschnitte später in Tocquevilles $D e$ la démocratie en Amérique - dessen Titel bereits dieses Amerika-Bild mit seiner Verklammerung ,Amerikas' mit der Demokratie einführt - in aller Deutlichkeit vorgeführt. Wir sind noch immer im neunten Kapitel des dritten Bandes:

Ich kann nicht damit übereinstimmen, Amerika von Europa zu trennen, trotz des Ozeans, der beide voneinander scheidet. Ich betrachte das Volk der Vereinigten Staaten als einen Teil des englischen Volkes, damit beauftragt, die Wälder der Neuen Welt auszubeuten, während der Rest der Nation, der über mehr Muße verfügt und sich weniger um die materiellen Sorgen des Lebens kümmern muss, sich dem Denken überlassen und den menschlichen Geist in alle Richtungen entwickeln kann.

Die Lage der Amerikaner ist daher völlig außergewöhnlich, und es steht zu glauben, dass kein demokratisches Volk sich jemals in einer solchen Situation befinden wird. Sein gänzlich puritanischer Ursprung, seine ausschließlich kommerziellen Sitten, das Land selbst, welches es bewohnt und seine Intelligenz vom Studium der Wissenschaften abzulenken scheint, die Literatur und die Künste; die Nachbarschaft Europas, die ihm erlaubt, sie alle nicht zu studieren, ohne dadurch doch in die Barbarei zurückzufallen; tausend spezielle Gründe, von denen ich nur die wichtigsten herausarbeiten konnte, mussten auf eine sehr eigene Weise den amerikanischen Geist in der Sorge um die rein materiellen Dinge formen. Die Leidenschaften, die Notwendigkeiten, die Erziehung, die Umstände, alles scheint in der Tat dazu beizutragen, dass sich der Bewohner der Vereinigten Staaten der Erde zuneigt. Allein die Religion lässt ihn von Zeit zu Zeit flüchtige Blicke zerstreut gen Himmel richten. Hören wir daher auf, alle demokratischen Nationen in der Figur des amerikanischen Volkes zu sehen, und versuchen wir, sie endlich mit ihren eigenen Zügen wahrzunehmen. ${ }^{8}$

In dieser längeren Passage wird deutlich, dass im Panorama einer transatlantischen Entwicklung eine neue geokulturelle und -politische Einheit in Entstehung begriffen ist, welche uns heute, nach einem langen 20. Jahrhundert, in den ersten Jahrzehnten des 21. Jahrhunderts mehr denn je wohlvertraut ist: Es handelt sich um die Einheit des Westens und vielleicht mehr noch einer transatlantischen angelsächsischen Welt. Wie stark unterscheidet sich doch dieses Bild von jenem, das sich bei Simón Bolívar und so vielen anderen ausdrückte, die im Ringen

8 Tocqueville, Alexis de: De la démocratie en Amérique, Bd. III, S. 70 f. 
um die hispanoamerikanische Unabhängigkeit den unverbrüchlichen Hass auf Spanien predigten. Im Gegensatz zu dieser Situation Lateinamerikas scheint der US-amerikanische Unabhängigkeitskampf gegen die britische Kolonialmacht weniger schmerzhafte Spuren hinterlassen zu haben.

In Tocquevilles Darstellung erscheinen die USA noch nicht als die Denkfabrik, als der Think Tank des Westens oder gar als Hort der Wissenschaften, sondern vielmehr als ein Land, in welchem man von den Fortschritten in den Wissenschaften und Künsten woanders profitiert. Denn gedacht wird noch immer woanders: in England, in Frankreich, kurzum im alten Europa. Das neue Bild der USA als Denkfabrik und wissenschaftlichem Motor des Okzidents wird sich erst in der zweiten Hälfte des 20. Jahrhunderts herauskristallisieren und in seiner Entstehungszeit noch einmal - vorerst ein letztes Mal - unter dem Sputnik-Schock erzittern. Was in dieser Passage aber sehr deutlich wird, ist die intime Verbundenheit dieses - und keines anderen - Amerika mit der westlichen Welt. Auch hier wird wieder offenkundig, dass die Kultivierung der Wälder von keinen Indianern, keinen anderen Bewohnern, den ,eigentlichen' Amerikanern - zumindest laut Tocqueville - geleistet wird. Es ist, als hätten wir es mit einer mittelalterlichen Landnahme in Europa zu tun, wo es keine vorherige Bevölkerung gegeben hätte.

Ein zweiter Punkt wird ebenfalls sehr deutlich zum Ausdruck gebracht. Er betrifft die feste Verbindung des Bildes der Vereinigten Staaten von Amerika mit dem schieren Materialismus. Dies wird eine Vorstellung sein, die das gesamte 19. Jahrhundert dominieren sollte und noch in Auffassungen der hispanoamerikanischen Modernisten von José Martí bis Rubén Darío erscheinen wird: der Glaube an einen spirituellen und geistig aufgeweckten Süden, welcher dem materialistischen und utilitaristischen Norden des Kontinents entgegenstünde. Insbesondere José Enrique Rodó hatte die USA in seinem im Jahre 1900 veröffentlichten Essay Ariel fast ausschließlich als die calibaneske Variante eines kruden Materialismus verstanden, der es - gemäß der Shakespeare'schen Triade Prospero-Ariel-Caliban - die immateriellen, geistigen und kulturellen Werte eines mit Ariel verbandelten lateinischen Amerika entgegenzustellen gelte. Das Gegenbild, mit dem der Uruguayer Rodó hier an der Wende zum 20. Jahrhundert spielt, ist im Verlauf des 19. Jahrhunderts entstanden und zu einem Gemeinplatz des lateinamerikanischen Selbstverständnisses geworden. Tocquevilles Werk gehört zweifellos zu den Schriften, die diesem zweigeteilten Bild der Amerikas wesentliche Aspekte hinzugefügt und es zugleich popularisiert haben.

Als weiterer Punkt wird schließlich neben der Tatsache, dass die Religion in den USA eine wichtige Rolle spiele und die einzig wirklich geistige, da geistliche Beschäftigung der Bewohner dieser Landstriche darstelle, darauf hingewiesen, dass man die demokratische Entwicklung nicht mit jener der USA verwechseln 
sollte. Dieser Hinweis zielt darauf, dass sich Tocqueville durchaus dagegen zur Wehr setzt, die gesamte Zukunft der demokratischen Länder und Völker - wie er sich ausdrückt - in der Gegenwart der USA und damit im Materialismus verankert zu sehen. Er erkennt zwar in den USA das Modell künftiger Entwicklung, weist zugleich aber darauf hin, dass dieses Modell aus einzigartigen Bedingungen entstanden sei, welche es nirgendwo anders gebe und daher - im aktuellen Beamtendeutsch gesagt - ein Alleinstellungsmerkmal aufweise. Die Vereinigten Staaten von Amerika zeigen laut Tocqueville an, wie sich die Demokratien des Westens entwickeln werden, aber es bestehe Hoffnung - so dürfen wir den französischen Geschichtsphilosophen begreifen -, dass sich mit Blick auf die Wissenschaften, aber auch auf Literatur und Künste nicht automatisch der krude Materialismus mit der Demokratie verbinden und durchsetzen werde. Tocqueville erblickt daher in Europa ein künftiges demokratisches Gegenmodell zu den Vereinigten Staaten, eine erhoffte Horizontlinie, die sich nach dem Zweiten Weltkrieg innerhalb des politischen Rahmens der kommenden Europäischen Union in der Tat verwirklicht hat, heute aber wieder in Gefahr ist.

Amerika im Sinne der USA aber zeigt letztlich die sich beschleunigende historische Bewegung an, die von einem unabhängig gewordenen, demokratischen und riesigen Land ausgeht, in ihm mit allen Auswirkungen nach außen spürbar wird. Die Zukunft ist - wie bereits betont - radikal offen, zugleich aber auch von ständigen Veränderungen und Wechseln geprägt, welche einen anderen Lebensrhythmus vorgeben. Schauen wir uns diese auch literarisch sehr überzeugend gestaltete Passage, ebenfalls aus dem dritten Teil von De la démocratie en Amérique, aufmerksam an:

In dem Maße, wie die Kasten verschwinden, wie die Klassen sich einander annähern, wie die Menschen sich tumultartig miteinander vermischen, wie die Gewohnheiten, die Gebräuche, die Gesetze sich verändern, wie neue Tatsachen auftauchen, wie neue Wahrheiten ins Licht gerückt werden, wie althergebrachte Meinungen verschwinden und wie andere an ihre Stelle treten, wird das Bild einer idealen und doch stets flüchtigen Perfektion sich dem menschlichen Geiste präsentieren.

Beständige Veränderungen werden sich dann in jedem Augenblicke unter den Augen jedes Menschen zeigen. Die einen verschlechtern seine Lage, und er wird nur zu gut verstehen, dass ein Volk oder ein Individuum, so gebildet es auch sei, keinesfalls unfehlbar ist. Die anderen verbessern sein Schicksal, und er schließt daraus, dass der Mensch im Allgemeinen mit der unbegrenzten Fähigkeit des Perfektionierens ausgestattet ist. Seine Rückschläge lassen ihn erkennen, dass nichts sich schmeicheln darf, das absolut Gute entdeckt zu haben; seine Erfolge werden ihn beflügeln, pausenlos fortzufahren. So tendiert der Mensch, immer auf der Suche, hinstürzend, sich wieder erhebend, oftmals enttäuscht, aber niemals entmutigt, ohne Unterlass zu jener unermesslichen Größe, welche er am Ende eines langen Weges erkennen kann und den die Menschheit noch durchlaufen muss.

Man sollte nicht glauben, wie viele Fakten sich ganz natürlich von dieser philosophischen Theorie herleiten, der zufolge der Mensch unbegrenzt perfektibel ist, und wie wunderbar 
der Einfluss ist, den sie selbst auf jene ausübt, welche sich allein mit dem Handeln und niemals mit dem Denken beschäftigten, zugleich aber ohne deren Kenntnis ihre Handlungen daran ausrichten. ${ }^{9}$

Es ist beeindruckend, wie Alexis de Tocqueville hier in einer raschen, fast atemlosen Satzfolge und in einem Rhythmus, der die rastlose Bewegung und die ständige Beschleunigung des von ihm Berichteten widerzugeben versucht, die Vorwärtsbewegungen von Menschen und eines ganzen Volkes, ja aller westlichen Demokratien und selbst der gesamten Menschheit plastisch darzustellen sucht. Es ist in seinen Augen ein sich ständig nach vorne orientierender, immer wieder fallender, aufstehender, weiter vorstürmender Mensch, der zwar keine kontinuierliche Bewegung, doch nur eine einzige Bewegungsrichtung kennt: vorwärts! Wie philosophisch und stilistisch einflussreich derartige Passagen waren, werden wir spätestens bei unserem Blick auf die Essays des hispanoamerikanischen Modernismo erkennen: Insbesondere José Martí und José Enrique Rodó knüpfen an diese Prosa und die mit ihr verbundene Vorstellungswelt an. Doch beeindruckend ist nicht zuletzt, dass Tocqueville nur von einem einzigen Weg der Menschheit spricht, alle Menschen folglich auf diesen Weg bezieht, auf dem selbstverständlich die europäischen oder europäisch geprägten Völker vorangehen.

Das zentrale Philosophem, das hinter all diesen ineinander verwickelten Gedankengängen steckt, ist das einer grenzenlosen Perfektibilität. Der Neologismus „perfectibilité““ war von Jean-Jacques Rousseau - den wir nicht von ungefähr an den Beginn unserer Vorlesung gestellt hatten - gleichsam in die philosophierende Welt gesetzt worden. Jenseits jeglichen Rousseauismus' aber wird in diesen Argumentationen die unendliche Fähigkeit des Menschen, sich ständig neu zu vervollkommnen, auf ein bestimmtes Gesellschaftsmodell übertragen. Wenige Worte später wird von einem Matrosen berichtet, der auf die Frage, warum die Amerikaner Schiffe bauten, die schon nach relativ kurzer Zeit verschrottet werden müssten, antwortet, dass die Fortschritte in der Schifffahrtstechnik so rasch vor sich gingen, dass es keinen Sinn mehr mache, dauerhaft verwendbare Schiffe zu bauen, insofern ständig neue Technologien derartige Schiffe rasch veralten ließen. So finden wir von diesem US-amerikanischen Matrosen in den Worten Tocquevilles bereits vorformuliert, was in späteren Zeiten das Wegwerf-Credo einer Gesellschaft des kapitalistischen Massenkonsums werden würde; ein Credo, das noch heute ebenso für unsere Automobile wie unsere Smartphones gilt.

Was ursprünglich einmal auf die Perfektibilität des Menschen und seine Fähigkeit sich weiterzuentwickeln abgezielt hatte, wird hier gleichsam zum

9 Tocqueville, Alexis de: De la démocratie en Amérique, Bd. III, S. 63 f. 
Handlungsprinzip eines demokratischen Volkes erklärt, das nach diesem Philosophem wie nach einem Gesetz des Handelns agiere. Zugleich wird das Philosophem der Perfektibilität auf das Wirtschaftssystem übertragen und avanciert zur Richtschnur einer Ökonomie, welche ständig neue Produkte hervorbringt, weil diese vorgeblich weit besser als ihre Vorgänger seien. Das mag in einigen Fällen zutreffen, in den weitaus überwiegenden aber stimmt es nicht. Auch auf dieser Ebene - das wird deutlich - wird die materialistische Dimension dieser Entwicklung betont; doch zugleich werden die großen Ideale der Menschheitsgeschichte beschworen. Alexis de Tocquevilles Demokratie in Amerika zeigt auf, wie sich die westlichen Demokratien insgesamt entwickeln werden. Und diese Entwicklung ist eine der Beschleunigung: einer Beschleunigung mit Unfällen, gewiss! Insofern verkörpert - wie wir schon sahen - die Entwicklung der demokratischen Vereinigten Staaten von Amerika eine Entwicklung in der Zukunft der demokratischen Völker Europas.

Alexis de Tocqueville war keineswegs der einzige, der sich einer philosophisch untermauerten Weltgeschichte annahm und in ihr eine Zukunft zu erkennen glaubte, in der Europa und die USA, nicht aber im eigentlichen Sinne der Rest Amerikas oder auch der ,Rest' der Weltgeschichte vorkamen. Doch es gab Gegenbeispiele: Die Herausforderung durch den nicht touristisch folklorisierten und zoologisierten ,Anderen', durch das in seiner kulturellen Vielfalt wahrgenommene und anerkannte ,Andere' wirkte für einen Philosophen, Kultur- und Naturforscher wie Alexander von Humboldt gerade im Bereich des Denkens stets fruchtbar, etwa auf dem Gebiet der Philosophie. Im selben historischen Zeitraum, am 30. Mai 1837, gestand der Weltreisende und Schriftsteller, der in Berlin nicht nur bei Carl Ritter, sondern auch bei Hegel Vorlesungen hörte, seinem Freund Varnhagen von Ense:

Hegel's geschichtliche Studien werden mich besonders interessiren, weil ich bisher ein wildes Vorurtheil gegen die Ansicht hege, dass die Völker, ein jedes, etwas repräsentiren müssen; dass alles geschehen sei, „damit erfüllet werde“ was der Philosoph verheißt. Ich werde aufmerksam lesen, und gern von meinem Vorurtheile zurückkommen. ${ }^{10}$

Bereits am 1. Juli 1837 aber meldete Humboldt in der für ihn typischen ironischen Diktion an Varnhagen:

10 Briefe von Alexander von Humboldt an Varnhagen von Ense aus den Jahren 1827 bis 1858. Nebst Auszügen aus Varnhagen's Tagebüchern und Briefen von Varnhagen und Andern an Humboldt. [Hg. von Ludmilla Assing.] Leipzig: F.A. Brockhaus 1860, S. 43. 
Ein Wald von Ideen ist freilich für mich in jenem Hegel, dem Gans so meisterhaft den Karakter seiner großen Individualität gelassen hat, aber für einen Menschen, der, wie ich, insektenartig an den Boden und seine Naturverschiedenheit gebannt ist, wird ein abstraktes Behaupten rein falscher Thatsachen und Ansichten über Amerika und die indische Welt freiheitraubend und beängstigend. Dabei verkenne ich alles das Großartige nicht. ${ }^{11}$

Doch in einer kurzen Nachschrift kann sich Humboldt doch nicht ganz des offenen Spotts enthalten: „Mein Leben habe ich recht schlecht eingerichtet, ich thue alles um recht früh stupide zu werden. Ich thäte gern „Verzicht auf das europäische Rindfleisch,“ das Hegel S. 77 so viel besser als das amerikanische fabelt, und lebte neben den schwachen kraftlosen (leider 25 Fuß langen) Krokodilen." ${ }^{12}$ Diese auf die im 18. Jahrhundert von Buffon, de Pauw, Raynal und vielen anderen sowie dann von Hegel fortgeführte Degenerationsthese, die alles in Amerika als kränklich und schwächlich ansah, musste dem stets empirisch verankerten und denkenden Geist Alexander von Humboldts gründlich missfallen. Lange zuvor hatte er sich bereits gegen jene Thesen von Cornelius de Pauw gewandt, ${ }^{13}$ die noch Jahrzehnte später - und von der Hegel-Forschung weitestgehend unbemerkt - dem deutschen Philosophen als Grundlagen seines weltphilosophischen Denkens dienten. Nur am Rande darf ich Sie darauf verweisen, dass dieses Hegel'sche „Fabulieren“ von kleinen, schwächlichen Krokodilen - nicht ohne eine leichte Verfälschung des Zitats - zum Gegenstand einer künstlerischen Installation mit dem Titel El cocodrilo de Humboldt no es el cocodrilo de Hegel geworden ist. ${ }^{14}$

Die Humboldt'sche Kritik an Hegel ist in vielerlei Hinsicht repräsentativ für seine eigene, stets empirisch fundierte Vorgehensweise. Sie zielt einerseits auf die mangelnde faktenbezogene Basis verabsolutierter Behauptungen sowie auf den Mangel an eigener Welt-Erfahrung, die Humboldt nicht nur gegen Hegel, sondern auch gegen Buffon, Raynal, Kant, Schelling und viele andere Philosophen und Welt-Deuter ins Feld hätte führen können und überwiegend auch führte. Andererseits richtet sie sich auch gegen die Systemhaftigkeit und immanente Teleologie

11 Ebda., S. 44.

12 Ebda., S. $44 \mathrm{f}$.

13 Vgl. Ette, Ottmar: „Not just brought about by chance“: reflections on globalization in Cornelius de Pauw and Alexander von Humboldt. In: Studies in Travel Writing (Nottingham) XV, 1 (February 2011), S. 3-25.

14 Vgl. die photographische Wiedergabe der 25 Fuß langen Installation El cocodrilo de Humboldt no es el cocodrilo de Hegel (Das Krokodil Humboldts ist nicht das Krokodil Hegels) des kolumbianischen Künstlers José Alejandro Restrepo; sie findet sich in der Alexander von Humboldt gewidmeten schönen Sondernummer der Zeitschrift Humboldt (Bonn) 126 (1999), S. 16 f. 
philosophischer wie wissenschaftlicher Systeme generell. Dies erklärt die Enttäuschung Humboldts angesichts jener Vorlesungen Hegels, von denen er sich weitaus mehr versprochen hatte.

In Hegels Vorlesungen über die Philosophie der Weltgeschichte, die Humboldt durchaus aufmerksam zur Kenntnis genommen hatte, mag es die fehlende empirische Basis von Überlegungen wie der folgenden zur Aufgabe der Philosophie gewesen sein, an denen sich Humboldts Wissenschaftskonzeption und sein Verständnis einer Untersuchung der Weltgeschichte rieben. Wir erkennen im folgenden Zitat durchaus Aspekte, die wir schon in Tocquevilles Überlegungen durchschimmern sahen, auch wenn der Franzose anders als Hegel auf die empirische Begründung seiner Argumentation achtete. Hören wir also Hegel in etwa so, wie der deutlich ältere Humboldt ihn gehört haben mag:

Die Philosophie hat es nur mit dem Glanze der Idee zu tun, die sich in der Weltgeschichte spiegelt. Aus dem Überdruß an den Bewegungen der unmittelbaren Leidenschaften in der Wirklichkeit macht sich die Philosophie zur Betrachtung heraus; ihr Interesse ist, den Entwicklungsgang der sich verwirklichenden Idee zu erkennen, und zwar der Idee der Freiheit, welche nur ist als Bewußtsein der Freiheit. Dass die Weltgeschichte dieser Entwicklungsgang und das wirkliche Werden des Geistes ist, unter dem wechselnden Schauspiele ihrer Geschichten, - dies ist die wahrhafte Theodicee, die Rechtfertigung Gottes in der Geschichte. Diesen Gang des Weltgeistes Ihnen zu entwickeln, ist mein Bestreben gewesen. ${ }^{15}$

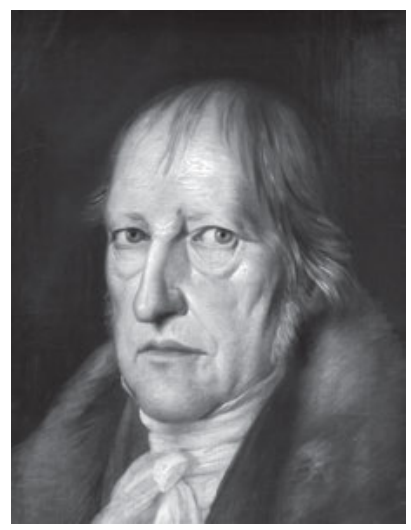

Abb. 42: Georg Wilhelm Friedrich Hegel (Stuttgart, 1770 - Berlin, 1831).

Alexander von Humboldt, der ganz konkret zu den von Hegel hier am Ende seiner Vorlesung angesprochenen Studenten gehört haben könnte, mochte es beim

15 Hegel, Georg Wilhelm Friedrich: Vorlesungen über die Philosophie der Weltgeschichte. Hamburg: Verlag Felix Meiner 1988, Bd. IV, S. 938. 
Gedanken an diesen Weltgeist, wie sehr er auch aus antiken Quellen schöpfte, etwas mulmig geworden sein. Nicht die ,Einpassung‘ vorhandenen Materials in vorgefertigte Denksysteme, sondern die lebendige Beziehung zwischen Analyse und Synthese, zwischen Datensammlung und Generalisierung, zwischen partikulärer Beobachtung und stets vorläufig bleibender Schlussfolgerung, die immer auf einer vergleichenden Vorgehensweise basierte, bildeten die Grundlage für die Humboldt'sche Wissenschaft. Und dies auch, wenn ihm die Idee der Freiheit, wiewohl in einem anderen Sinne als demjenigen Hegels, beständig vor Augen stand. Die Idee der Freiheit war dem Humboldt'schen Denken wie auch seiner auf Reisen basierenden Lebenspraxis intrinsisch.

Für uns aber ist mehr als interessant, dass die Position Hegels sich für lange Zeit durchsetzen sollte; eine Position, in der Amerika im Sinne der Vereinigten Staaten erstmals als Kontinent der Zukunft annonciert wurde, zugleich aber eben jener Reduzierung Amerikas auf die USA entsprach, der wir bereits bei Alexis de Tocqueville begegnet waren. Dies ist für die geistesgeschichtliche Entwicklung des 19. Jahrhunderts, aber zugleich bis hinein in unsere Gegenwart grundlegend. Georg Friedrich Wilhelm Hegel war nicht zufällig Teil des Disputs um die Neue Welt - und sein Standort war im Grunde noch immer der eines Cornelius de Pauw. Daher ordnete er sich eindeutig auf der Seite der meisten europäischen Philosophen des 18. Jahrhunderts ein, mithin derjenigen Cornelius de Pauws, Guillaume-Thomas Raynals oder William Robertsons. Sein Geschichtsbild zeigt dies ganz eindeutig; und ich möchte an dieser Stelle gerne einmal die entsprechende Passage aus Hegels einflussreichen philosophischen Überlegungen zitieren:

Die Welt wird in die Alte und Neue geteilt, und zwar ist der Namen der neuen daher gekommen, weil Amerika und Australien uns erst spät bekannt geworden sind. Aber diese Weltteile sind nicht nur relativ neu, sondern überhaupt neu, in Ansehung ihrer ganzen physischen und geistigen Beschaffenheit. [...] Von Amerika und seiner Kultur, namentlich in Mexiko und Peru, haben wir zwar Nachrichten, aber bloß die, dass dieselbe eine ganz natürliche war, die untergehen mußte, sowie der Geist sich ihr näherte. Physisch und geistig ohnmächtig hat sich Amerika immer gezeigt und zeigt sich noch so. [...] Die Inferiorität dieser Individuen in jeder Rücksicht, selbst in Hinsicht der Größe, gibt sich in allem zu erkennen; nur die ganz südlichen Stämme in Patagonien sind kräftigere Naturen, aber noch ganz in dem natürlichen Zustande der Roheit und Wildheit. ${ }^{16}$

16 Hegel, Georg Wilhelm Friedrich: Vorlesungen über die Philosophie der Geschichte. Auf der Grundlage der Werke von 1832-1845 neu edierte Ausgabe. Redaktion von Eva Moldenhauer und Karl M. Michel. 10. Aufl. Frankfurt a.M.: Suhrkamp 2012 (= Hegel Werke 12), S. 107 f. 
Diese Sätze entstammen nicht der Feder eines Philosophen der Aufklärung, nicht den Werken eines Robertson, eines de Pauw oder Raynal, sondern den Vorlesungen des an der neugegründeten Berliner Universität lehrenden Georg Wilhelm Friedrich Hegel, der im selben Berlin wie Alexander von Humboldt lebte. Er wiederholt noch Jahrzehnte später dieselben Phrasen und haltlosen Beschuldigungen, die er der europäischen Aufklärungsliteratur entnommen hatte. Gleichzeitig finden sich in diesen und weiteren Formulierungen all jene Stereotype wieder, die seit der Entdeckung der sogenannten ,Neuen Welt' in Europa gesammelt, auf diese projiziert und im 18. Jahrhundert nochmals gebündelt wurden. Hegel war in diesem Kontext lediglich ein Schallverstärker, der ungeachtet jeglicher empirischen Basis jene Elemente übernahm, die in sein weltphilosophisches System passen wollten. Dass er damit die Stimme Humboldts übertönte, weil er die europäischen Vorurteile aufgriff und wirksam verbreitete, ist eine Tatsache, die wir aus heutiger Perspektive zwar bedauern mögen, die den „Gang der Weltgeschichte“ aber befeuerte.

Hegels weltgeschichtliche Betrachtungen gipfeln in seiner bekannten Sentenz „Amerika ist somit das Land der Zukunft“17 nur deshalb, um Amerika mit den USA gleichzusetzen. Im selben Atemzug werden die ,eigentlichen‘ Amerikaner sowie die Bewohner des überwiegenden Teils des Kontinents aus der Philosophie der Weltgeschichte verbannt, da sich diese allein „mit dem, was weder nur gewesen ist noch erst nur sein wird“ und letztlich allein „mit der Vernunft“18 beschäftige. In dieser Philosophie ist für die von Humboldt beschriebene Welt kein Platz: „Nordamerika“ und „Südamerika“ stehen einander unvermittelt gegenüber; und der letztgenannte Teil des Doppelkontinents, dem Hegel ausdrücklich (aber geographisch unkorrekt) auch Mexiko zurechnet, kann weder durch seine letztlich geleugnete kulturelle Vergangenheit noch durch seine Gegenwart - die „auf militärischer Gewalt“ beruhe und „ein fortdauernder Umsturz“ sei ${ }^{19}$ - Anspruch auf Erlösung aus seiner weltgeschichtlichen Verdammung erheben. Die Weltgeschichte kann aus Hegels Sicht ohne Verlust auf diesen Teil der Welt verzichten ganz so, wie es vor Hegel schon de Pauw mit dem größten Teil der Menschheit getan hatte.

Diese weltgeschichtliche Argumentationslinie dürfte Karl Marx und mehr noch Friedrich Engels dazu verleitet haben, Hegel in diesem Falle einmal nicht „auf den Kopf zu stellen“, sondern im Sinne ihres Fortschrittsdenkens praktisch anzuwenden. So fragt Engels in einem ursprünglich im Januar 1848 veröffent-

17 Ebda., S. 114.

18 Ebda.

19 Ebda., S. 111. 
lichten Beitrag mit Blick auf den 1846 bis 1848 erfolgreich gegen Mexiko geführten nordamerikanischen Expansionskrieg, ob „es etwa ein Unglück“ sei, „dass das herrliche Kalifornien den faulen Mexikanern entrissen ist, die nichts damit zu machen wußten“. ${ }^{20}$ Der Beutezug der USA in der sogenannten „Guerra de rapiña“ wird von Friedrich Engels damit aus weltpolitischer Sicht vollauf gerechtfertigt, da für ihn die Vereinigten Staaten von Amerika anders als der gesamte ,Süden“ des Kontinents eine globalgeschichtliche Rolle übernommen hatten. Und er setzt seine rhetorische Frage, die ein ganz bestimmtes Projekt der Moderne skizzierte, unerschütterlich fort:

Dass die energischen Yankees durch die rasche Ausbeutung der dortigen Goldminen die Zirkulationsmittel vermehren, an der gelegensten Küste des stillen Meeres in wenig Jahren eine dichte Bevölkerung und einen ausgedehnten Handel konzentrieren, große Städte schaffen, Dampfschiffsverbindungen eröffnen, eine Eisenbahn von New York bis San Francisco anlegen, den Stillen Ozean erst eigentlich der Zivilisation eröffnen, und zum dritten Mal in der Geschichte dem Welthandel eine neue Richtung geben werden? Die „Unabhängigkeit“ einiger spanischer Kalifornier und Texaner mag darunter leiden, die „Gerechtigkeit“ und andre moralische Grundsätze mögen hie und da verletzt sein; aber was gilt das gegen solche weltgeschichtliche Tatsachen ${ }^{21}$

In diesen und ähnlichen Passagen wird Hegels Rede von Amerika als dem „Land der Zukunft“ ausschließlich mit Blick auf die USA in die Vision eines weltgeschichtlichen Prozesses umgesetzt, zu dessen Protagonist auf dem amerikanischen Kontinent allein jene Vereinigten Staaten werden konnten, die erst nur Kalifornien und Texas, bald aber ganz Mexiko dem nun neu definierten Weltgeist überantworteten. Auch wenn die Unterschiede in der Zielrichtung eklatant sein mögen, erweist sich Engels - und mit ihm auch Marx - als Vertreter eines teleologischen Denkens, für das Weltgeschichte nur unilateral als eine einzige Einbahnstraße denkbar ist. Die ersten auf dieser Einbahnstraße in der Entwicklungsgeschichte der Menschheit mussten dabei selbstverständlich die Europäer (einschließlich ihrer amerikanischen ,Ableger') sein.

Auch das Humboldt'sche Weltbewusstsein ${ }^{22}$ ist keineswegs frei von teleologischen Elementen, vor einer Absolut-Setzung des Weltgeistes - wie auch immer dessen Dialektik gedeutet werden mag - aber dadurch geschützt, dass es Mensch

20 Engels, Friedrich: Der demokratische Panslawismus. In: Marx, Karl / Engels, Friedrich: Werke. Bd. 6. Berlin: Dietz Verlag 1959, S. 273.

21 Ebda., S. $273 \mathrm{f}$.

22 Vgl. hierzu Ette, Ottmar: Weltbewusstsein. Alexander von Humboldt und das unvollendete Projekt einer anderen Moderne. Mit einem Vorwort zur zweiten Auflage. Weilerswist: Velbrück Wissenschaft 2020. 
und Natur empirisch fundiert aufeinander bezieht, kulturelle Differenz jenseits der Zivilisation zu denken erlaubt und die künftige welthistorische Entwicklung als einen multipolaren Prozess begreift. Dieser sollte laut Alexander von Humboldt zum Wohlstand und Nutzen aller Völker beitragen.

Mit Blick auf die geschichtsphilosophische Orientierung am Mittelmeer, an den dortigen Raumverhältnissen und dem von dort ausgehenden weltgeschichtlichen Prozess ergeben sich zwischen Hegels und Humboldts Konzeptionen sehr wohl Parallelen, welche zweifellos auf die gemeinsame Nähe zu Vorstellungen Goethes und zur ,Weimarer‘ Deutung der griechischen Antike verweisen. Für Humboldt wie für Hegel stand der Mittelmeerraum im Fokus aller welthistorischen Betrachtungen und bildete jenen Kreuzungspunkt, an dem sich die Prozesse der Weltgeschichte neu perspektivierten. Doch hätten diese Deutungsversuche bei Humboldt niemals zur Behauptung einer aus den Raumverhältnissen abgeleiteten Totalität geführt, die den Ausschluss anderer Welten - und damit der Welt des ,Anderen', des Nicht-Geistes - mit sich bringen musste.

Denn für Hegel ist allein die Alte Welt „Schauplatz der Weltgeschichte“; ${ }^{23}$ selbst das „weite östliche Asien ist vom Prozesse der Weltgeschichte entfernt“"24 und umso mehr musste es daher für ihn der weltphilosophisch vernachlässigbare ,Rest' von Amerika sein:

Amerika ist in zwei Teile geteilt, welche zwar durch eine Landenge zusammenhängen, die aber nur einen ganz äußerlichen Zusammenhang bildet. Die Alte Welt dagegen, welche Amerika gegenüberliegt und von demselben durch den Atlantischen Ozean getrennt ist, ist durch eine tiefe Bucht, das Mittelländische Meer, durchbrochen. Die drei Weltteile derselben haben ein wesentliches Verhältnis zueinander und machen eine Totalität aus. Ihr Ausgezeichnetes ist, dass sie um das Meer herumgelagert sind und darum ein leichtes Mittel der Kommunikation haben. [...] Das Mittelmeer ist so das Herz der Alten Welt, denn es ist das Bedingende und Belebende derselben. Ohne dasselbe ließe sich die Weltgeschichte nicht vorstellen, sie wäre wie das alte Rom oder Athen ohne das Forum, wo alles zusammenkam. ${ }^{25}$

Sie ersehen deutlich aus diesen Passagen: Das sind geokulturelle und geopolitische Überlegungen, die direkt mit der Debatte um die Amerikas verbunden sind und deutlich eine geostrategisch-weltgeschichtliche Komponente aufweisen! Die ganze Weltgeschichte - oder in seinen Worten: ihre „Totalität“ - dreht sich also bei Hegel um das Mittelmeer, wobei er den deutschsprachigen Regionen durchaus

23 Hegel, Georg Wilhelm Friedrich: Vorlesungen über die Philosophie der Geschichte, S. 115.

24 Ebda.

25 Ebda. 
eine besondere Bedeutung zuerkennt. In Hegels Weltgeschichte gibt es aus der hier gewählten Perspektive freilich keinen Platz, wo wirklich alles in allen Zeiten zusammenkommt: Die Weltgeschichte bleibt als solche in Bewegung.

Georg Wilhelm Friedrich Hegels, Friedrich Engels' und auch Alexander von Humboldts Entwürfe sind zweifellos Projekte einer europäischen Moderne, doch unterscheidet sich Humboldts Projekt deutlich von allen anderen dadurch, dass es wissenschaftlich umfassender fundiert, grundlegend komparatistisch (und damit zwischen verschiedenen kulturellen Areas vergleichend) angelegt und nicht monokulturell, sondern zumindest interkulturell ausgerichtet ist. Dies gilt es gerade dann nicht zu vergessen, wenn uns manches in seinem Denken heute bekannt, bisweilen sogar selbstverständlich vorkommt. Doch wir werden uns ja noch mit Alexander von Humboldts Denken näher auseinandersetzen ...

Kehren wir an dieser Stelle aber zu unserem Ausgangspunkt, gleichsam der Zeitreise zurück, und fragen wir uns danach, ob es denn Reisen in die südlichen Teile der amerikanischen Hemisphäre gab, die ebenfalls als Zeitreisen aufgefasst werden können oder derartige vektorielle Elemente enthalten! Wir hatten ja bereits verschiedentlich gesehen, dass sich bei Reisen in den Süden oftmals wie in Alejo Carpentiers Los pasos perdidos - eine Reise in die Vergangenheit der Menschheit als Grundmuster abzeichnete. Lässt sich dies durch Belege aus zeitgenössischen Texten des 19. Jahrhunderts substanziell belegen?

Die europäischen Reisenden des 18. und wohl auch noch jene des 19. Jahrhunderts glauben sehr wohl an eine gemeinsame Zeit und Entwicklungsgeschichte der Menschheit, eine transhistorische Zeitachse also, auf die sich die von ihnen konstatierten verschiedenen Zeitebenen linear beziehen lassen. Bei einer derartigen Vorstellung wird die Zeitreise notwendigerweise zur Bewegung des Reisenden zwischen verschiedenen Stufen kultureller, historischer, ökonomischer und sozialer Entwicklung, unabhängig davon, ob diese Entwicklung positiv oder negativ eingefärbt, ob die Entwicklung folglich als Höherentwicklung oder als Degradation gelesen wird. Bei Jean-Jacques Rousseau etwa dominierte eindeutig eine Entwicklungslinie der Degradation ${ }^{26}$ in der Totalität menschheitsgeschichtlicher Entwicklung von einer Urzeit her, in welcher die Menschen in glücklicher Konvivenz miteinander friedlich zusammengelebt hätten. Erst später habe sich dann die Ungleichheit im weiteren Verlauf der Menschheitsgeschichte mehr und mehr etabliert.

26 Vgl. hierzu insbesondere Rousseau, Jean-Jacques: Diskurs über die Ungleichheit. Discours sur l'inégalité. Kritische Ausgabe des integralen Textes. Mit sämtlichen Fragmenten und ergänzenden Materialien und den Originalausgaben und den Handschriften neu ediert. Übersetzt und kommentiert von Heinrich Meier. Paderborn - München - Wien - Zürich: Ferdinand Schöningh 1984. 
Bei Autorinnen und Autoren des 19. Jahrhunderts dominiert nicht allein in der Reiseliteratur bei Bewegungen in außereuropäische Weltregionen insgesamt eine einheitliche Vorstellung vom Verlauf der Menschheitsgeschichte ${ }^{27}$ - und zwar unabhängig davon, ob sie (was seltener vorkam) einer Geschichte der Degradation oder (was deutlich häufiger existierte) einer Geschichte des ständigen Fortschritts den Vorzug gaben. Die Entdeckung voneinander unabhängiger, partikularer Zeiten gewinnt - soweit ich sehe - erst in der Reiseliteratur des 20. Jahrhunderts zunehmend an Raum.

Auch in Flora Tristans bereits erwähnter Reise nach Peru, in ihren Pérégrinations d'une paria, ist die Erfahrung der Zeitreise wiederholt gegenwärtig. Denn die Verfasserin des bis heute faszinierenden weiblichen Reiseberichts ${ }^{28}$ glaubt sich etwa ins europäische Mittelalter versetzt, als sie die Mysterienspiele im peruanischen Arequipa literarisch darstellt. Wählen wir als Beleg einen kurzen Textausschnitt aus dieser Phase ihrer Reisebewegung! Die zweibändige Originalausgabe erschien 1838 unter dem Titel Pérégrinations d'une paria 1833-1834 und entwickelt die folgende Sicht dieser ,Alterität‘:

Für mich, ein Kind des 19. Jahrhunderts und aus Paris kommend, war die Aufführung eines Mysterienspiels unter dem Portal einer Kirche und vor einer unermesslich großen Volksmenge etwas Neues; aber das lehrreiche Schauspiel waren die Brutalität, die grobe Kleidung, die Lumpen eben dieses Volkes, dessen extreme Unwissenheit, dessen dummer Aberglaube meine Einbildungskraft ins Mittelalter zurückführten. ${ }^{29}$

Notiert Flora Tristan auch sehr genau, wie schnell und mit nur wenigen Jahren Verzögerung die damalige französische Mode die Toilette der peruanischen Frauen diktiert, kommt sie doch nicht umhin, aus dem, was sie als Aberglauben bezeichnet, den Schluss zu ziehen, dass das peruanische Volk noch in seiner Kindheit $^{30}$ verharre und lange der Kirchenmacht ausgeliefert bleiben werde. Neben der Wahrnehmung einer Gleichzeitigkeit notiert sie demgemäß ebenfalls eine Ungleichzeitigkeit, wobei beide Zeitebenen in Peru nebeneinander bestehen. Doch auch wenn die Mode in diesem südamerikanischen Land ,auf dem Stand“ der neuesten französischen Entwürfe ist, so befinden sich zumindest Teile der Bevölkerung noch immer im Mittelalter, wodurch der Zeitpfeil der temporalen Vektorisierung deutlich auf derselben Entwicklungsgeschichte Europas in die Vergangenheit, gleichsam in das Mittelalter Europas weist. Der literarische Bezugs-

27 Vgl. Ette, Ottmar: ReiseSchreiben, S. 458-570.

28 Vgl. hierzu das Flora Tristan gewidmete Kapitel in ebda., S. 543-555.

29 Tristan, Flora: Pérégrinations d'une paria. Bd. 1, S. 303.

30 Ebda., S. 280: „So sind die Völker in der Kindheit.“ 
punkt für Floras Darstellung eines Mysterienspiels blieb freilich nicht ungenannt: Die Erzählerin selbst verweist auf Victor Hugos Notre-Dame de Paris, ${ }^{31}$ das nur wenige Jahre zuvor erschienen war. Literatur und literarische Erfahrung prägen die Lebenserfahrungen der Reisenden und konstruieren jenes Hintergrundwissen, das zur Einordnung der vor Ort aufgenommenen Reiseeindrücke führt.

Alexis de Tocquevilles Reise von 1831 in die USA führte den Franzosen in die Zukunft, Flora Tristans Reise von 1833 nach Peru die Französin dagegen in die Vergangenheit. Spätestens in der ersten Hälfte des 19. Jahrhunderts entsteht aus europäischer Sicht ein zweigeteiltes Amerika, dessen Wirkmächtigkeit auf die europäische Imagination zweifellos bis zum heutigen Tage anhält. Bei dem Franzosen wie der Französin jedoch wird das ,Andere', die Zeit des ,Anderen' auf dieselbe, von Europa aus gedachte Zeitachse und deren Chronologie bezogen ein interessantes ,chassé-croisé“, das noch dadurch an Reiz gewinnt, dass beide höchst unterschiedlichen, an der Vergangenheit beziehungsweise der Zukunft orientierten Wertvorstellungen anhingen. Wäre der eine dem rechtskonservativen, monarchistischen politischen Spektrum zuzuordnen, so ließe sich die Französin zweifellos dem sozialistischen Lager zurechnen, für das sie sich nach ihrer Rückkehr nach Frankreich für den Rest ihres höchst aktiven Lebens aktiv engagierte.

Wir bewegen uns in beiden Reiseberichten auf der vierten Dimension, der Dimension der Zeit. Diese beinhaltet die Koexistenz, das Ineinander-Spielen verschiedener Zeitachsen und Zeitvorstellungen unter Einbeziehung der damit verbundenen (geographischen, kulturellen oder politischen) Räume. Die Konfrontation verschiedener Zeitebenen trägt wesentlich zum Reiz und zur Attraktivität des literarischen Reiseberichts bei. Darüber sollten wir aber nicht vergessen, dass sich beide Europäer ungeachtet ihrer politischen Orientierungen an derselben Entwicklungsachse einer einzigen Menschheitsgeschichte orientierten, die für alle von ihnen bereisten ethnischen Gruppen oder Völker ebenso in Europa wie außerhalb dieses kleinen Teiles der Erdoberfläche gültig sein musste.

Die hier untersuchte Zeitdimension stellt mit Blick auf Amerika eine zentrale Scharniersituation dar, die im Verlauf des ersten Drittels des 19. Jahrhunderts klar auf eine neue Sichtweise der Amerikas umgestellt hat, die der unsrigen, also heute noch aktuellen, sehr vertraut ist. Es ist - wenn Sie so wollen - die eigentliche Amerika-Sicht der europäischen Moderne, welche lange Zeit für die Moderne schlechthin gehalten wurde. Diese Erfindung eines zweigeteilten Amerika, diese aus europäischer Perspektive erfundene Findung der beiden Amerikas als einander fremd gegenüberstehende Einheiten mit unterschiedlichen Zeitvektoren,

31 Ebda., S. 304. 
beherrscht das Denken nicht zuletzt in Wirtschaft und Politik, aber auf vielen anderen Ebene bis heute.

Aus diesem Blickwinkel wird deutlich, dass es zumindest für das industrialisierte Westeuropa, bald aber auch für den gesamten europäischen Raum nur noch ein einziges Amerika gab, das zählen konnte: das Amerika der Zukunft, von dem Hegel sprach und das sich Engels auch im imperialen Ausgreifen nach Süden erträumte. Wie diese Expansion des Imperiums tatsächlich dann auch militärisch umgesetzt wurde, werden wir am Beispiel der hispanoamerikanischen Reaktionen auf das Eingreifen der USA in den spanisch-kubanischen Krieg im Jahre 1898 noch sehen. Doch noch ist die „New Steel Navy“ weit.

Und so lassen Sie uns auf die Tatsache kommen, dass die Zweiteilung des amerikanischen Kontinents keineswegs die erste diskursive Zweiteilung war, die das 19. Jahrhundert vornahm! Denn ihr ging eine andere, in Europa selbst vorgenommene Zweiteilung voraus, welche aus transarealer Sicht wie die Zweiteilung Amerikas von größter Relevanz für unsere Sichtweise der kulturellen wie literarischen Entwicklung sein muss. Diese ,Bifurkation' aber verbindet sich mit dem Namen einer Frau, deren Einbeziehung für den Fortgang unserer Analyse der Romantik unverzichtbar scheint und die zugleich - ebenso wie Flora Tristan von größter Bedeutung für die Entfaltung eines weiblichen Selbstbewusstseins am Übergang zum 19. Jahrhundert ist. 